\title{
Formation of Superhard State of the TiZrHfNbTaYN Vacuum-Arc High-Entropy Coating
}

\author{
V. M. Beresnev ${ }^{a}$, O. V. Sobol' ${ }^{b}$, A. A. Andreev ${ }^{c}$, V. F. Gorban'd , S. A. Klimenko ${ }^{e}$, *, \\ S. V. Litovchenko ${ }^{a}$, D. V. Kovteba ${ }^{c}$, A. A. Meilekhov ${ }^{b}$, A. A. Postel'nik ${ }^{b}$, U. S. Nemchenko ${ }^{a}$, \\ V. Yu. Novikov ${ }^{\prime}$, and B. A. Maziilin ${ }^{a}$ \\ ${ }^{a}$ Karazin Khar'kiv National University, Khar'kiv, Ukraine \\ ${ }^{b}$ National Tekhnical University Khar'kiv Polytechnic Institute, Khar'kov, Ukraine \\ ${ }^{c}$ National Science Center Khar'kiv Institute of Physics and Technology, \\ National Academy of Sciences of Ukraine, Khar'kiv, Ukraine \\ ${ }^{d}$ Frantsevich Institute of Materials Science Problems, \\ National Academy of Sciences of Ukraine, Kiev, Ukraine \\ ${ }^{e}$ Bakul Institute for Superhard Materials, \\ National Academy of Sciences of Ukraine, Kiev, Ukraine \\ $f_{\text {Belgorod National State research university, Belgorod, Russia }}$ \\ *e-mail: atmu@meta.ua \\ Received March 3, 2017
}

\begin{abstract}
Complex studies of the formation of the superhard state in the TiZrHfNbTaYN vacuum-arc high-entropy coating were carried out. Based on the approach of the structural surface engineering, the regularities of the formation of the triads composition-structure-physico-mechanical properties depending on the supplied potential displacement are established. It is shown that the increase of $U_{b}$ at the formation of a coating leads to a decrease of the relative content of a light (Ti) and increase of a heavy (Ta, Hf) metal components, which is determined by radiationally stimulated processes in a near-surface region at the deposition. The formation of the single-phase state (based on the fcc of metal lattice) in the range $U_{b}$ from -50 to $-250 \mathrm{~V}$ and revealed the formation of the preferred orientation of the crystallites with the axis [111], which is perpendicular to the growth plane. The increase of the perfection of the texture with the [111] axis with increasing $U_{b}$ is accompanied with an increase of the coatings hardness, which makes it possible to achieve the superhard state $(H=40.2 \mathrm{GPa})$ at $U_{b}=-250 \mathrm{~V}$.
\end{abstract}

DOI: $10.3103 / \mathrm{S} 1063457618020041$

Keywords: high-entropy coating, displacement potential, composition, structure, properties, texture, superhard state.

In the course of operation of the machine components and cutting tools their surface layers undergo strong mechanical, thermal, and chemical action. In the majority of cases the loss of a serviceability of products from structural materials occurs through their damages due to the wear, erosion, corrosion, which initiate in the surface layer. Because of this different methods of the layer modification are effective. The required results may be achieved at the deposition of multifunctional coatings [1-3]. The use of such coatings gives rise to the increase of the tools efficiency and durability of machine components. Owing to high mechanical characteristics and thermal stability, the protective layer may implement functional properties of products under severe conditions of the contact loading for a long time. Except for a high hardness, the protective coating should provide a low friction coefficient, a good adhesion to a substrate, stability to oxidation and wear resistance. Most often to produce coatings the vacuum-arc methods of deposition are used [4, 5].

In several recent years to increase the thermal stability of materials the concept of high entropy (or as in a number of publications they are called multiunit) alloys [6] and coatings based on their nitrides gain their development [7-9]. As a result of the intensive mixing the entropic contribution of the alloys components increases and ensures the possibility of the formation of a solid solution with a simple structure [10]. The principal singularity of high entropic alloys is the presence of the heterogeneous atoms of the elements in the lattice of the solid solution. Having different electronic structures, sizes and thermodynamic properties, in the crystal lattice of the substitutional solid solution they lead to its distortion [11, 12]. This facilitates a considerable solid solution strengthening. A decreased free energy of high-entropy alloys ensures the stability of the solid solution at high temperature [13-15]. 
In the present study to produce coatings with a high hardness, low friction coefficient, good adhesion to the substrate, as the basic elements of high-entropy alloys transition metals ( $\mathrm{Ti}, \mathrm{Zr}, \mathrm{Hf}, \mathrm{Nb}, \mathrm{Ta}, \mathrm{Y}$ ) with high heat of the nitride formation were used [16].

The aim of the work was the determination of the possibility of the use of the vacuum-arc deposition to produce materials with high mechanical properties from compositions containing strong nitride-forming components at their deposition under different potentials of the displacement.

\section{SAMPLES AND INVESTIGATION PROCEDURE}

Coatings were deposited using vacuum-arc method on a Bulat 6 plant. Previously the cathode of the needed composition was prepared by the vacuum-arc refining of a multicomponent mixture of the pure metals powders. The crystallized ingot was taken out from a crystallizer, turned over and placed into the crystallizer again. To obtain the maximum homogeneous structure, the solution was repeatedly melted; the procedure was repeated 7 times. An ingot as a cylinder (the diameter was $45 \mathrm{~mm}$ and height $30 \mathrm{~mm}$ ) was extracted from the crystallizer and soldered to a titanium cathode with a hard solder. Thus, the cathodes-targets based on the Ti$\mathrm{Zr}-\mathrm{Nb}-\mathrm{Hf}-\mathrm{Y}-\mathrm{Ta}$ system were made and used to produce coatings. Nitrogen was the reaction gas. The deposition parameters are given in Table 1. This contributes to a considerable solid solution strengthening.

Table 1. Technological parameters of the TiZrHfNbTaYN coating deposition of the ( $I_{\mathrm{d}}$ is the arc current, $I_{\mathrm{f}}$ - current of the cathode focusing, $U_{b}$ is the displacement potential, $p$ is the pressure in a chamber, $H V_{0.2}$ is the Vickers hardness at the load of $0.2 \mathrm{kG}$ )

\begin{tabular}{c|c|c|c|c|c}
\hline Series & $I_{\mathrm{d}}, \mathrm{A}$ & $I_{\mathrm{f}}, \mathrm{A}$ & $U_{b}, \mathrm{~B}$ & $p$, Torr & $H V_{0.2}, \mathrm{GPa}$ \\
\hline 1 & 110 & 0.5 & -50 & $2 \times 10^{-3}$ & 18.7 \\
2 & & & -100 & & 30.1 \\
3 & & & -200 & & 32.6 \\
4 & & & -250 & & 40.2 \\
\hline
\end{tabular}

As the substrates for a deposition of coatings the samples $(R a=0.09 \mu \mathrm{m})$ of steel $12 \mathrm{X} 18 \mathrm{H} 9 \mathrm{~T}(15 \times 15 \times$ $2.5 \mathrm{~mm}$ ) were used, the deposition time was $1.5 \mathrm{~h}$, the coating thickness $\sim 8.0 \mu \mathrm{m}$.

The surface morphology, fractographs of the fractures, friction trails were studied using a scanning electron microscope FEI Nova NanoSEM 450. The study of the elemental composition of coatings was performed by analyzing spectra of the characteristic X-ray radiation, which was taken using the energy dispersive spectrometer of the PEGASUS system of the EDAX Company.

The phase-structure state was investigated on a DRON-3M diffractometer in the $\mathrm{CuK \alpha}$ radiation. For the monochromatization of the registrated radiation a graphite monochromator was used which was placed in a second beam (before a detector). The investigations of the phase composition, structure (texture, substructure) were made using the traditional procedures of the X-ray diffractometry by analyzing location, intensity and forms of profiles of diffraction reflexes. Substructural characteristics average microdeformation $<\varepsilon>$ and size of the crystallites $L$ ) were defined using the approximation method in two orders of reflexes from the $\{111\}$ planes [17].

The hardness of coatings was measured with the hardness meter of the DM 8 model according to the micro-Vickers method at the indentation load $0.2 \mathrm{~N}$.

The determination of adhesion and cohesion stability, durability to scratching and clearing of the fracture mechanism of coatings were carried out using a scratch-tester Revetest (CSM Instruments SA, Switzerland). On the surface of the coating the scratches were brought by a diamond spherical indenter of the Rockwell C type with a rounded radius of $200 \mu \mathrm{m}$ at the uninterruptedly increasing load. The power of the acoustic emission signal, friction coefficient and the depth of the indenter penetration as well as the value of the normal load were simultaneously registered. To obtain reliable results, three scratches were applied to the surface of each sample with a coating. The tests were carried out under the following conditions: the indentation load increased from 0.9 to $70 \mathrm{~N}$, the rate of the indenter shift was $1 \mathrm{~mm} / \mathrm{min}$, the scratch length $10 \mathrm{~mm}$, the rate of the load supplied $6.91 \mathrm{~N} / \mathrm{min}$, the frequency of the signal discreteness- $60 \mathrm{~Hz}$, the power of the acoustic emission-to $9 \mathrm{~dB}$.

In the result of the tests the minimum (critical) loads were defined: $L_{C 1}$ is the corresponding to the onset of the indenter penetration into the coating, $L_{C 2}$ - the appearance of the first crack, $L_{C 3}$-appearance of the 
accumulation of cracks, $L_{C 4}$ - spalling of some regions of coating, $L_{C 5}$-cleavage of the coating or its plastic abrasion to the substrate.

\section{RESULTS AND DISCUSSION}

To establish the linkage in the composition-structure-properties triad, at first the elemental composition of coatings was investigated. The characteristic energy dispersive spectra are given in Fig. 1 and the resultant data of the elemental composition in Table 2. As is seen, the displacement potential affects the redistribution of the heaviest (Hf, Ta) and lightest (Ti) metal atoms components of a coating in the greatest degree. Thus, with the increasing $U_{b}$ from -50 to $-250 \mathrm{~V}$ the contents of the $\mathrm{Hf}$ and $\mathrm{Ta}$ in coatings increases from 13.68 to 18.54 at $\%$ and from 8.65 to 11.50 at $\%$, respectively. For the metal element having the least weight (Ti) with the increasing $U_{b}$ from -50 to $-250 \mathrm{~V}$ the content decreases from 12.34 to 6.95 at $\%$. The changes with the increase of $U_{b}$ obtained for the elements with medium weight ( $\mathrm{Nb}$ and $\mathrm{Zr}$ ) are insignificant. The revealed regularities of the changes of the elemental composition with increase of $U_{b}$ indicate that the composition of the metallic atoms determines the secondary ballistic scattering from the growth surface $[10,11]$. The contents of light gas atoms of nitrogen decrease with the increase of $U_{b}$ as well as the content in the coating of titanium atoms with the strong coupling Ti-N (a great negative heat of the formation is $336 \mathrm{~kJ} /$ mole [18]). The results obtained provide the possibility to state that the process of the nitride formation and development of stable metal-nitrogen complexes occur after the selective secondary sputtering from the growth surface, i.e., the process of the formation of stable nitrides occurs directly in the near-surface layers of growth.

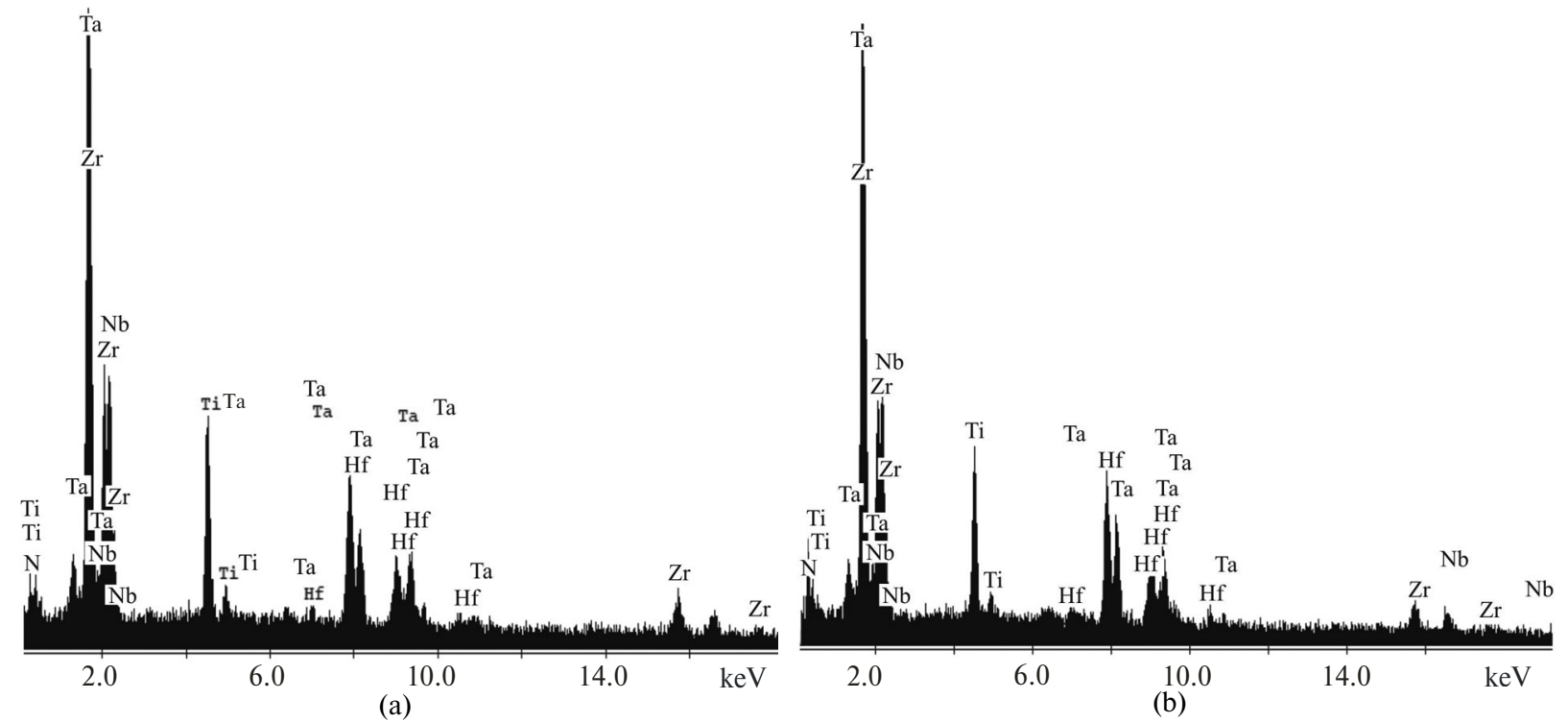

Fig. 1. Energy dispersive spectra from coatings taken at $U_{b}=-50$ (a) and -200 (b) V.

It should be noted that the revealed regularities of the direction of the elemental composition at the selective scattering in the process of the deposition broaden the possibility of the structural engineering by varying $U_{b}$.

Table 2. Elemental composition of the coating, at \%

\begin{tabular}{c|c|c|c|c|c|c}
\hline Series & $\mathrm{Ti}$ & $\mathrm{Nb}$ & $\mathrm{Zr}$ & $\mathrm{Ta}$ & $\mathrm{Hf}$ & $\mathrm{N}$ \\
\hline 1 & 12.37 & 12.50 & 14.95 & 8.65 & 13.68 & 37.84 \\
2 & 10.99 & 12.77 & 14.89 & 9.24 & 14.39 & 37.72 \\
3 & 9.73 & 12.60 & 14.85 & 10.80 & 16.43 & 36.31 \\
4 & 6.95 & 12.93 & 14.30 & 11.50 & 18.54 & 35.77 \\
\hline
\end{tabular}

The influence of the displacement potential affects also the structural state of the coating. Figure 2 gives the diffraction spectra from the coating produced at the $U_{b}$ from -50 to $-250 \mathrm{~V}$. It is seen that in all cases the single-phase state formation in the coating takes place. The displacement potential essentially affects the con- 
ditions of the growth of crystallites, identifying the preferred orientation of planes. At $U_{b}=-50 \mathrm{~V}$ there forms practically a random-orientation state (see Fig. 2, spectrum 1). At the $U_{b}=-100 \mathrm{~V}$ the intensity of diffraction peaks of the systems of $\{111\}$ planes (see Fig. 2, spectrum 2), which defines the preferred orientation of crystallites with the [111] axis, perpendicular to the growth surface. At the $U_{b}$ increases to $-200 \mathrm{~V}$ (see Fig. 2, spectrum 3) and $-250 \mathrm{~V}$ (see Fig. 2, spectrum 4) the relative intensity of the $\{111\}$ peaks becomes stronger, which points to the fact that the more perfect texture [111] forms. In this case the displacements of the peaks locations to the side of the lowest angles takes place (is shown by the arrow in Fig. 2), which at the $\theta-20$-scheme of the survey corresponds to the intensification of the action of the compression macrostress in a coating [19].

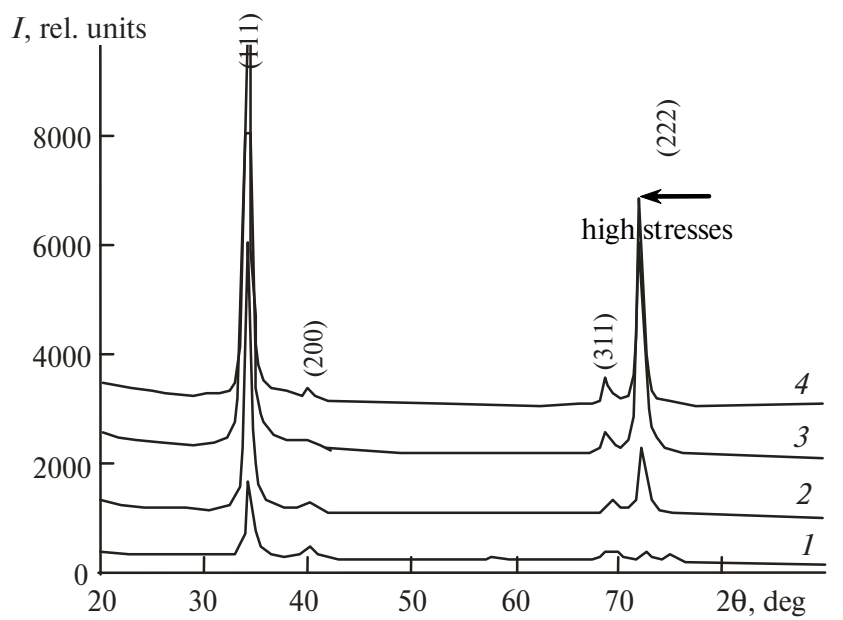

Fig. 2. Regions of the diffraction spectra of coatings deposited at $U_{b}=-50(1),-100(2),-200(3),-250$ (4) V.

At the substructure level in the maximum measure the displacement potential affects the changes of the microdeformation. The medium size of the crystallites at all $U_{b}$ remains sufficiently large $(110-170 \mathrm{~nm})$ and changes nonmonotonic (Table 3). The reason for a considerable microdeformation of crystallites at the comparatively small $U_{b}$ is the low mobility of atoms and, accordingly, low ability to the relaxation of deformation defects in crystallites $[9,20]$. A supply of a great negative displacement potential at the deposition stimulates an increase of the medium energy of the precipitable particles, which defines great possibilities to relaxation of the structure defects under the formation of coating. With this bound the relatively low microdeformation in crystallites of coatings produced at a high $U_{b}$ (see Table 3 , series 3,4 ).

As it was already mentioned, the most important characteristics for the majority of the fields of the coating applications are their hardness, adhesion strength, and friction coefficient, determining the wear resistance.

Table 3. Substructural characteristics of coatings — the mean microdeformation $<\varepsilon>$ and sizes of crystallites $L$

\begin{tabular}{c|c|c}
\hline Series & $<\varepsilon>, \%$ & $L, \mathrm{~nm}$ \\
\hline 1 & 1.3 & 150 \\
2 & 0.67 & 120 \\
3 & 0.43 & 110 \\
4 & 0.48 & 170 \\
\hline
\end{tabular}

The results of the measuring microhardness given in Table 1 show that with increasing $U_{b}$ (and accordingly, at the structural level with the increase of the degree of the texture perfection [111]) the coating hardness increases up to the superhard state $H=40.2 \mathrm{GPa}$ at $U_{b}=-250 \mathrm{~V}$.

The investigation of the mechanical properties of coatings was also carried out by a scratch-testing. In this case the process of the destruction of coatings at the scratching with a diamond indenter one may divide into several stages (Fig. 3). At the first stage (the region to the $L_{C 1}$ ) the monotonous penetration of the indenter into the coating. In this case the coating exhibits the essential resistance to the indenter penetration, the friction coefficient nonmonotonically increases (at the expense of the coating roughness), the acoustic emission (AE) signal remains practically invariable. Further at the increasing load (the region from $L_{C 1}$ to $L_{C 2}$ ) the $\mathrm{AE}$ amplitude level and friction coefficient start to change, whereas the depth of the indenter penetration 
increases nonessential. The further increase of the load (the region from $L_{C 2}$ to $L_{C 3}$ ) the level of the AE amplitude and the friction coefficient begin to change and the indenter penetration depth increases nonessentially. The subsequent increase of (the load of the region from $L_{C 2}$ to $L_{C 3}$ ) leads to the nonmonotonic change of the friction coefficient at the practically uncontaminated depth of the indenter penetration. To avoid the mistakes at the determination of the friction coefficient caused by the determining of the appearance of cracks at the

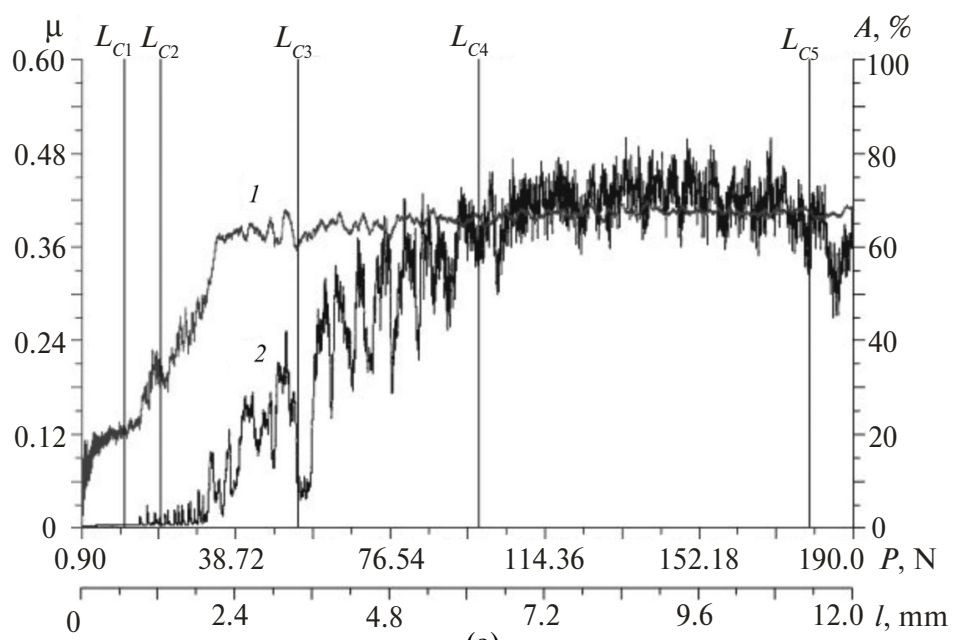

(a)

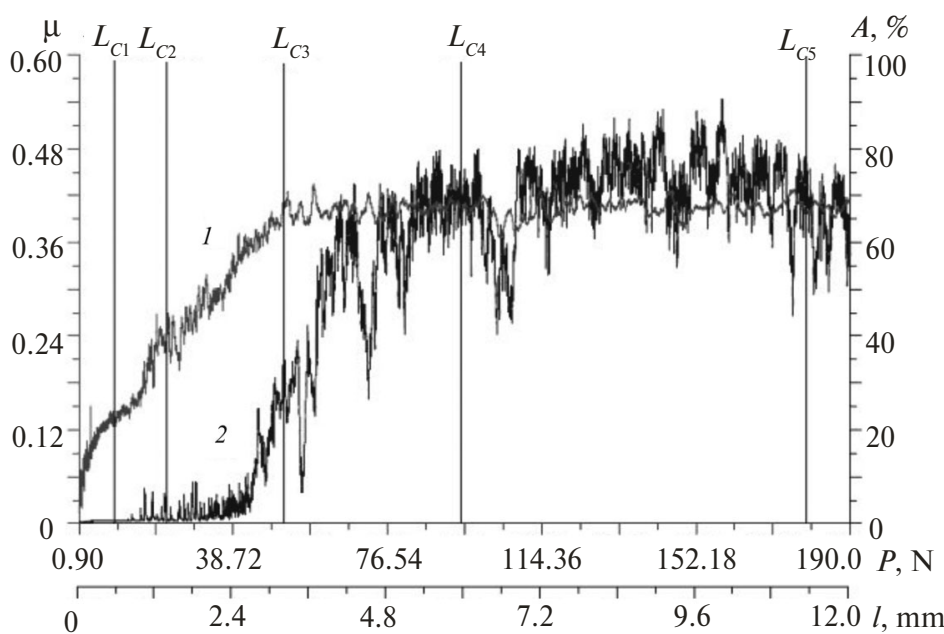

(b)

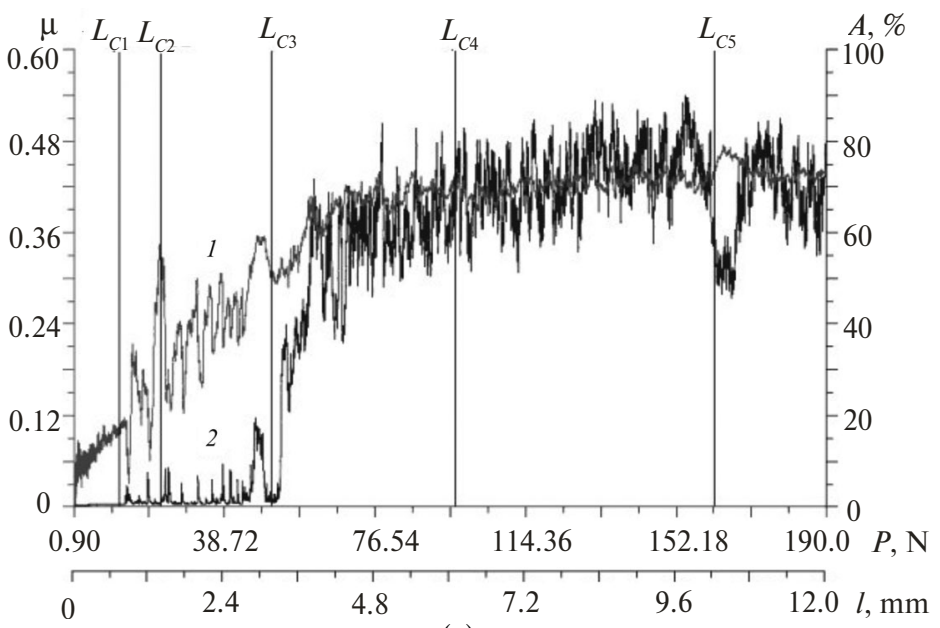

(c)

Fig. 3. Average values of the friction coefficient $\mu$ (spectrum 1, left scale) and amplitudes of acoustic emission $A$ (spectrum 2 , right scale) depending on the length of a pass and the applied force at the penetration of a diamond indenter for coatings, taken at $U_{b}=-50$ (a), -100 (b), -250 (c) V. 
increase of the indentation load, the analysis of this parameter was made on the regions of tests to the formation of the accumulation of cracks and destruction of the cracks accumulation and destruction of coatings, i.e., on the regions to $L_{C 3}$. For all samples of coatings the mean value found by this method is $0.38-0.40$. It should be noted that in coating produced at not high $U_{\mathrm{b}}=-50 \mathrm{~V}$ (see Fig. 3a), in the region from $L_{C 2}$ to $L_{C 3}$ the abrupt jump of the friction coefficient accompanied by the increase of peaks of acoustic emission. The reason for this is the relatively low hardness of coating (see Table 1), which as it follows from the analysis of friction tracks (Fig. 4a) corresponds to the regime of its plastic abrasion.

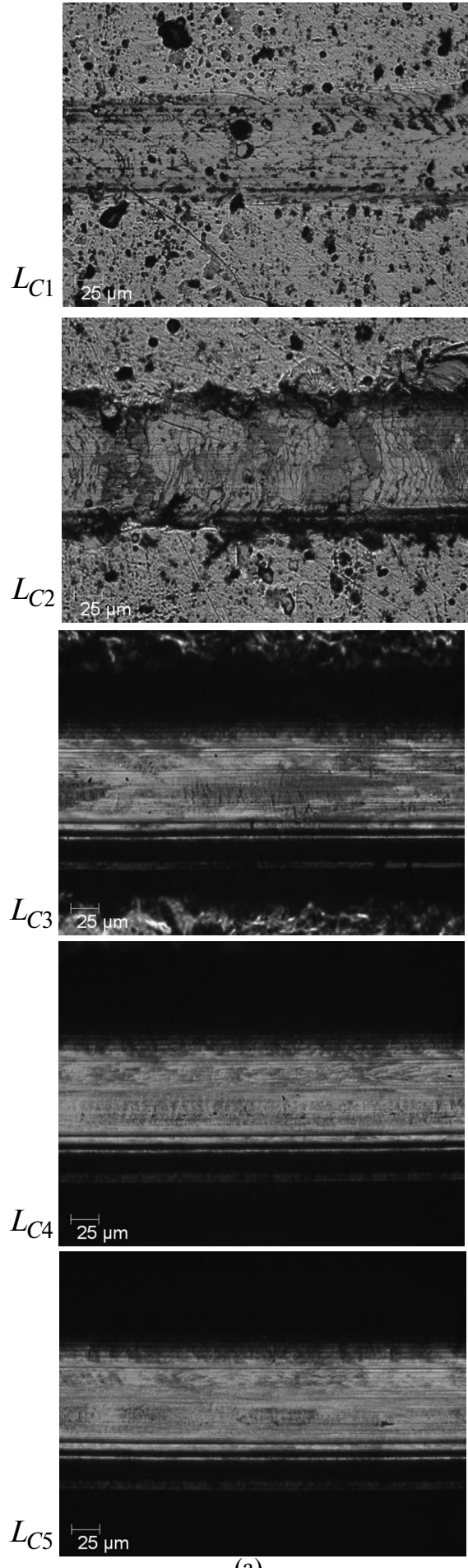

(a)
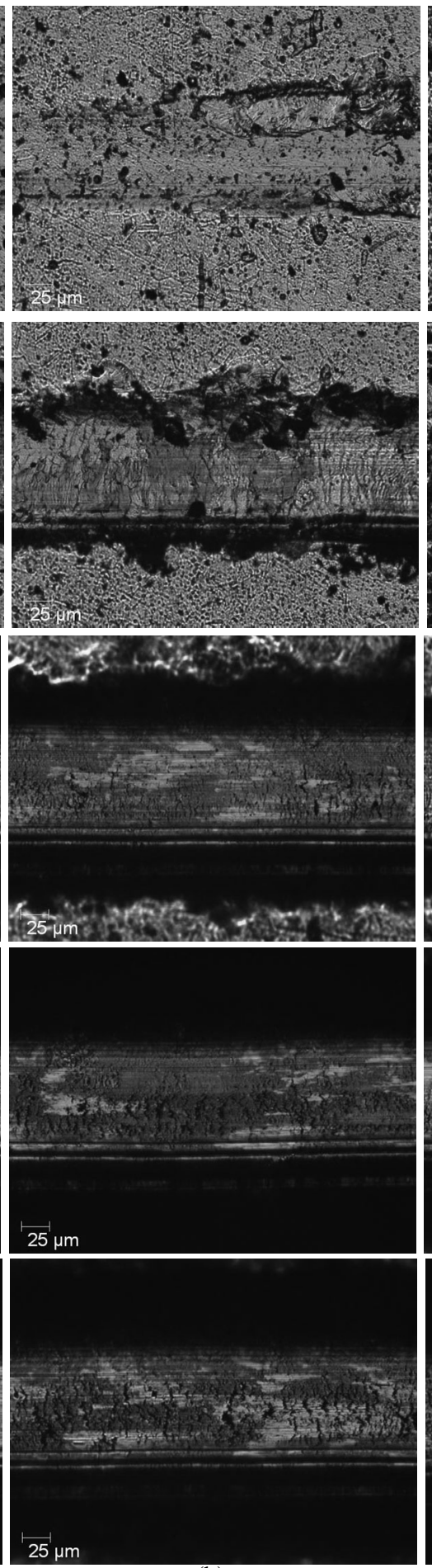

(b)
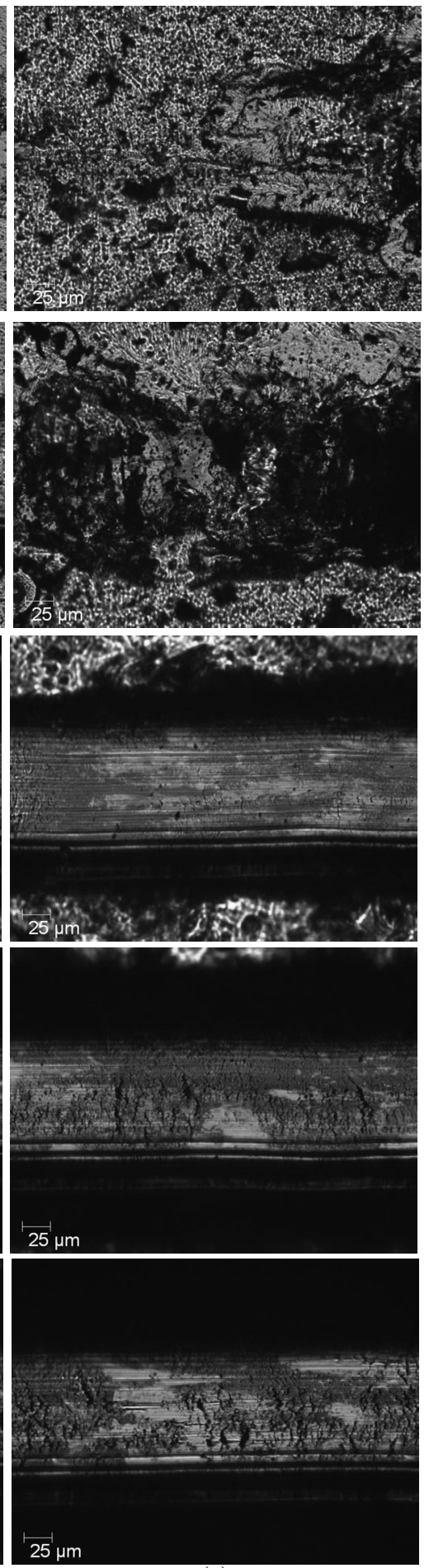

(c)

Fig. 4. Wear tracks in the field of the loads corresponding to critical points $L_{C}$ for coatings taken at the different $U_{b}=-50$ (a), $-100(\mathrm{~b}),-250(\mathrm{c}) \mathrm{V}$. 
In coatings produced at higher $U_{\mathrm{b}}$ at the region from $L_{C 2}$ to $L_{C 3}$ a smooth variation of the friction coefficient occurs at the low meaning of acoustic emission amplitude. This type of the dependence is the most expressed in Fig. $3 \mathrm{c}$ for a coating produced at the highest $U_{\mathrm{b}}=-250 \mathrm{~V}$, for which the hardness is the highest. In the regions from $L_{C 3}$ and further the even process of the abrasion is settled, that as evidenced the value close to the constant value of the friction coefficient.

The analysis of the wear track (see Fig. 4) allows visually characterize the processes occurring at the scratch-testing. It is seen that at the $L_{C 1}$ stage with increasing coating hardness (see Figs. 4a-4c) the wear degree decreases. The same effect is observed also in the regions to $L_{C 3}$.

In this case with the hardness increase the coating wear become more closely associated with the brittle fracture of the contact surfaces, which is seen from the increase of the unevenness on the friction tracks. Such a picture remains up to the critical wear at $L_{C 3}$ (see Fig. 4).

The obtained load values for the critical points $L_{C}$ are generalized in Table 4 . It is seen that at the initial regions the values of critical loads increases with the increasing coating hardness. The highest values of the $L_{C 1}$ $(12.32 \mathrm{~N})$ are obtained for series 4 with a hardness $40.2 \mathrm{GPa}$. However, at the higher loads the lower brittleness of less hard coatings of series 1 and 2 allow to attain high values of the adhesion strength (as a criterion of the adhesion strength the critical load $L_{C 5}[21]$ was used that leads to the destruction of the coating).

The highest value of the adhesion strength attained in coatings of series 1 and is $179.33 \mathrm{GPa}$, which is a high index [22].

Table 4. Critical loads for points $L_{C}$ of coatings

\begin{tabular}{c|c|c|c|c|c}
\hline Series & $L_{C 1}, \mathrm{~N}$ & $L_{C 2}, \mathrm{~N}$ & $L_{C 3}, \mathrm{~N}$ & $L_{C 4}, \mathrm{~N}$ & $L_{C 5}, \mathrm{~N}$ \\
\hline 4 & 12.32 & 22.83 & 50.56 surface & 96.51 & 169.9 \\
2 & 11.58 & 20.19 & 53.92 & 98.2 & 177.27 \\
1 & 9.57 & 22.45 & 51.04 & 94.54 & 179.33 \\
\hline
\end{tabular}

\section{CONCLUSIONS}

The complex research of high entropy nitride vacuum-arc coating produced on the base of the conditions of the surface structural engineering $[23,24]$ allowed establishing the regularities of the formation of the composition-structure-physico-mechanical properties triad depending on the supplied $U_{b}$.

It is established that the selective dispersion by atom at the formation of a coating leads to a decrease of the relative content of a light component (Ti) and to the increase of heavy components (Ta, Hf) at the increase of $U_{b}$.

For the multiunit vacuum-arc coating TiZrHfNbTaYN the formation of the single-phase state (on the base of the fcc of a metal lattice) is established over the whole examined $U_{b}$ range from -50 to $-250 \mathrm{~V}$.

The formation of the preferred orientation of crystallites with the [111] axis perpendicular to the plane of growth is revealed. The degree of the texture perfection increases with the increase of $U_{b}$. The growth of the texture perfection with the [111] axis is accompanied by the increase of the hardness of coatings, which allows at the increase $U_{b}=-250 \mathrm{~V}$ to attain the superhard state $(H=40.2 \mathrm{GPa})$.

A high hardness makes for low wear of a coating at low loads of the scratch-testing, but the highest value of the critical load of the destruction $L_{C 5}=179.33 \mathrm{GPa}$ is achieved in a less hard random-orientation coatings produced at $U_{b}=-50 \mathrm{~V}$.

\section{REFERENCES}

1. Modern tribology: Resume and prospects, Editor Frolov, K.V., Moscow: Publishing house LKI, 2008.

2. Pogrebnjak, A.D., Beresnev, B.M., Bondar, O.V., Abadias, G., Chartier, P., Postol'nyi, V.A., Andreev, A.A., and Sobol', O.V., The effect of nanolayer thickness on the structure and properties of multilayer TiN/MoN coatings, Technical Phys. Lett., 2014, vol. 40, no. 3, pp. 215-218.

3. Nanostructured coatings, Cavaleiro, A. and De Hosson, J. Th. M., Eds., New York: Springer-Verlag, 2006.

4. Roos, J.R., Celis, J.P., Vancoille, E., Veltrop, H., Boelens, S., Jungblut, F., Ebberink, J., and Homberg, H., Interrelationship between processing, coating properties and functional properties of steered arc physically vapor deposited (Ti, Al)N and (Ti, Nb)N coatings, Thin Solid Films, 1990, vol. 193-194, pp. 547-556. 
5. Sobol', O.V., Andreev, A.A., Grigoriev, S.N., Gorban, V.F., Volosova, S.N., Aleshin, S.V., and Stolbovoy, V.A., Physical characteristics, structure and stress state of vacuum-arc TiN coating, deposition on the substrate when applying high-voltage pulse during the deposition, Problems Atomic Sci. Technol., 2011, no. 4, pp. 174-177.

6. Dolique, V., Thomann, A.-L., Brault, P., Tessier, Y., and Gillon, P., Complex structure/composition relationship in thin films of $\mathrm{AlCoCrCuFeNi}$ high entropy alloy, Mater. Chem. Phys., 2009, vol. 117, no. 1, pp. 142-147.

7. Senkov, O.N., Scott, J.M., Senkova, S.V., and Miracle, D.V., Microstructure and room temperature properties of a high-entropy TaNbHfZrTi alloy, J. Alloys Compd., 2011, vol. 509, no. 20, pp. 6043-6048.

8. Pogrebnjak, A.D., Yakushchenko, I.V., Abadias, G.,Chartier, P., Bondar, O.V., Beresnev, V.M., Takeda, Y., Sobol', O.V., Oyoshi, K., Andreyev, A.A., and Mukushev, V.A., The effect of the deposition parameters of nitrides of high-entropy alloys (TiZrHfVNb) N on their structure, composition, mechanical and tribological properties, $J$. Superhard Mater., 2013, vol. 35, no. 6, pp. 356-368.

9. Azarenkov, N.A., Sobol', O.V., Beresnev, V.M., Pogrebnjak, A.D., Kolesnikov, D.A., Turbin, P.V., and Toryanik, I.N., Vacuum-plasma coatings based on multielement nitrides, Metallophyzika i Noveishie Tekhnologii, 2013, vol. 35, no. 8, pp. 1061-1084.

10. Sobol' O.V., Andreev, A.A., Gorban' V.F., Krapivka, N.A., Stolbovoi, V.A., Seryuk, I.V., and Fil'chikov, V.E., Reproducibility of the single-phase structural state of the multielement high-entropy $\mathrm{Ti}-\mathrm{V}-\mathrm{Nb}-\mathrm{Zr}-\mathrm{Hf}$ system and related superhard nitrides formed by the vacuum-arc method, Techn. Phys. Lett., 2012, vol. 38, no. 7, pp. 616-619.

11. Sobol', O.V., Structural engineering vacuum-plasma coating inter-stitial phases, J. Nano-Electron. Phys., 2016, vol. 8, no. 2, art. 02024.

12. Barmin, A.E., Sobol', O. V., Zubkov, A.I., and Mal'tseva, L.A., Modifying effect of tungsten on vacuum condensates of iron, Phys. Metals Metallography, 2015, vol. 116, no. 7, pp. 706-710.

13. Huang, P.K. and Yeh, J.W., Effects of substrate bias on structure and mechanical properties of (AlCrNbSiTiV)N coatings, J. Phys. D: Appl. Phys., 2009, vol. 42, no. 11, art. 115401.

14. Dolique, V., Thomann, A.-L., Brault, P., Tessier, Y., and Gillon, P., Thermal stability of AlCoCrCuFeNi high entropy alloy thin films studied by in-situ XRD analysis, Surf. Coat. Technol., 2010, vol. 204, pp. 1989-1992.

15. Tsai, M.-N., Wang, C.-W., Tsai, C.-W., Shen, W.-J., Yeh, J.-W., Can, J.-Y., Wu, W.-W., Thermal stability and performance of NbSiTaTiZr high-entropy alloy barrier for copper metalization, J. Electrochem. Soc., 2011, vol. 158, no. 11, pp. H1161-H1165.

16. Samsonov, G.V., Nitridy (Nitrides), Kiev: Nauk. Dumka, 1969.

17. Sobol', O.V., The influence of nonstoichiometry on elastic characteristics of metastable $\beta-\mathrm{WC}_{1-x}$ phase in ion plasma condensates, Techn. Phys. Lett., 2016, vol. 42, no. 9, pp. 909-911.

18. Samsonov, G.V., Pryadko, I.F., and Pryadko, L.F., Elektronnaya lokalizatsiya v tverdom tele (Electronic localization in a solid), Moscow: Nauka, 1976.

19. Pogrebnyak, A.D., Sobol', O.V., Beresnev, V.M., Turbin, P.V., Dub, S.N., Kirik, G.V., and Dmitrenko, A.E., Features of the structural state and mechanical properties of $\mathrm{ZrN}$ and $\mathrm{Zr}(\mathrm{Ti})-\mathrm{Si}-\mathrm{N}$ coatings obtained by ion-plasma deposition technique, Techn. Phys. Lett., 2009, vol. 35, pp. 925-931.

20. Sobol', O.V., Andreev, A.A, Gorban', V.F., Krapivka, N.A., Stolbovoi, V.A., Serdyuk, I.V., Fil'chikov, V.E., Reprocibility of the single-phase structural state of the multielement high-entropy $\mathrm{Ti}-\mathrm{V}-\mathrm{Zr}-\mathrm{Hf}$ system and related superhatrd nitride formed by the vacuum-arc method, Ibid., 2012, vol. 38, no. 7, pp. 616-619.

21. Valli, J., A review of adhesion test methods for thin hard coatings, J. Vac. Sci. Technol. A, 1986, vol. 4, no. 6, pp. 30073014.

22. Beresnev, V.M., Klimenko, S.A., Sobol', O.V., Grankin, S.S., Stolbovoi, V.A., Turbin, P.V., Novikov, V.Yu., Meilekhov, A.A., Litovchenko, S.V., Malikov, L.V., Effect of the deposition parameters on the phase-structure state, hardness, and tribological characteristics of $\mathrm{Mo}_{2} \mathrm{~N} / \mathrm{CrN}$ vacuum-arc multilayer coatings, J. Superhard Mater., 2016, vol. 38, no. 2, pp. 114-122.

23. Bell, T., Surface engineering, past, present and future, Surface Eng., 1990, vol. 1, pp. 31-40.

24. Morton, V.D., Wang, H., Fleming, R.A., and Zou, M., Nanoscale surface engineering with deformation-resistant core-shell nanostructures, Tribology Lett., vol. 42, no. 1, pp. 51-58. 